\title{
Study of remote experiment management based on Labview
}

\author{
Chunxi Zhao \\ Jilin Agricultural University,Changchun 130118,China \\ 10821395@qq.com
}

Keywords: Virtual network laboratory; OPC; virtual experiment; LabVIEW

\begin{abstract}
This article designs and exploits the multilayer distributed mixed virtual network laboratory (Virtual NetLab) based on LabVIEW, and presents a new scheme of data acquisition which combines LabVIEW with OPC technology. This scheme primly solves the laboratory's difficult problem of data collection. Moreover, the remote-client of Virtual NetLab is enriched by the LabVIEW's strong network function. The experiment results show that the results of the remote client experiment and the running results of the local experimental system are consistent. And the delay time is less than 1s. The function of remote synchronous experiment is realized. At the same time, it proves that the control algorithm has good control effect, which proves the availability of the virtual network lab and the good operation performance.
\end{abstract}

\section{Introduction}

With the rapid development of micro electronic technology and computer technology, virtual instrument (Instruments Virtual, VI) came into being. Virtual instrument technology is a revolution in the history of the development of modern instruments[1]. It has had an immeasurable impact on the development of science and technology and industrial production. At the same time, it also brings the dawn to the development of virtual laboratory. Virtual laboratory was structured based on network technology and virtual instrument technology. On the one hand, it can make the laboratory experiment teaching equipment keep its advanced, and closely follow the pace of the development of modern science and technology. And it can reduce the experimental cost and improve the experimental use rate. On the other hand, it can make students break the limitation of traditional experiment time and space. VI can complete a variety of experiments by remote computer, to obtain the same experience as the real experiment[2].

At present, most of the virtual network laboratory system is composed of 3 main functions: remote client, server and field equipment. Among them, the server is the core of the entire virtual network lab, which connects the remote client and the field control terminal[3]. The server is a bridge of data communication. LabVIEW provides the ability of a convenient access and a powerful real-time control as a virtual instrument development platform. A complete virtual laboratory was build by using LabVIEW. The virtual experiment instrument was developed with its own component, which is not only powerful, but also the appearance is the same as the real instrument[4]. Thus, LabVIEW become the first choice of development platform in virtual laboratory equipment.

\section{General architecture of Virtual Network Lab}

A complete industrial control virtual laboratory system is constructed by using the powerful graphical programming language and network technology of LabVIEW. The system is mainly composed of 3 parts: the field control terminal, the central server, the remote client[5]. The central server is the core of the system. On the one hand, the OPC technology is used to collect the experimental data of the field control data in real time; On the other hand, the field data is transmitted to the remote client.

In particular, the field control end of the system comprises a monitoring layer and a device layer. The device layer includes various types of heterogeneous control systems in the laboratory. Each control system is equipped with their monitoring layer, that is, OPC server. The central server based on LabVIEW achieved the connection with the site control by DataSocket technology. The 
DataSocket provides a data source or target that is the interface of the OPC server, which shield the complexity of the underlying TCP programming[6]. So, the underlying data acquisition problem of virtual network lab is solved. At the same time, LabVIEW remote panel technology builds the center server of the B/s system structure. The system achieved internet release function with built-in Server Web technology. The remote client can realize the access to the virtual network lab in the browser.

\section{Module structure of Virtual Laboratory}

With the aid of LabVIEW development environment, a complete industrial control virtual network laboratory system based on the Institute of automation laboratory is developed. The experiment module is added to the system by the way of the sub VI based on the idea of LabVIEW modular programming[7]. In each of the sub VI, the more complex functional modules are encapsulated into the next level of the sub VI. Such a design method, the system has a better flexibility and reusability, and is conducive to future maintenance and expansion. When there are new experimental projects, only the new experimental project is designed and VI is added to the main program. It can complete the whole system update or maintenance.

In the system, the server receives the request of the experiment and the experiment instruction. According to different experiment command, the system choose different experiment module. And the system collected the data and downloaded the experimental parameters through the corresponding data communication module. The final results were outputted after the completion of data processing. At present, the system has developed two kinds of experimental modules: based on the physical class and virtual simulation class. The field experiment is mainly based on the existing equipment of the Institute of automation laboratory[8]. It achieved the industrial process control system with OPC technology, including the DCS virtual liquid level/temperature control system, industrial Ethernet virtual liquid level/temperature control system and a series of process control experiment project.

\section{DCS process control experiment module}

The following describes the typical case in the field experiment module: the design flow of the liquid level control experiment of DCS process and the related experimental test. The experimental module is based on the DCS control network with local control. DCS control network is composed of SUPCON technology development limited JX 300X DCS to achieve. It communicates with the III QXLPC process control experimental device in real time, and it is used to exchange data and control parameters with the OPC server and the OPC client and the virtual server[9]. After the experimental data was processed by the center server, and were transmitted to the remote customer on the Internet by server Web. At the same time, the control parameters of the remote client can also be transferred to the DCS through the central server, which can realize the control of the process control system. The solution to this problem is the DataSocket technology of LabVIEW and Server Web in front of the communication problem. DataSocket technology enables the virtual laboratory to be well integrated with OPC technology, and realized the real-time collection of the field data; The Server Web technology achieved the user's remote control laboratory[10]. In the experiment, the liquid level of the boiler in the III QXLPC process control experiment device is selected as the controlled variable. This is a kind of nonlinear control system with pure time delay, and the mathematical model is difficult to establish precisely. And it is a very classical controlled object in the actual industrial production. It is difficult to meet the requirements of the system control performance with the conventional PID algorithm or the single control method. In order to obtain better control effect, this study developed a intelligent PID controller that was charged for controlled device[11]. An intelligent PID controller composed of a single neuron with self-learning and adaptive ability. The controller has an online self tuning function, and its structure is shown in Figure 1. 


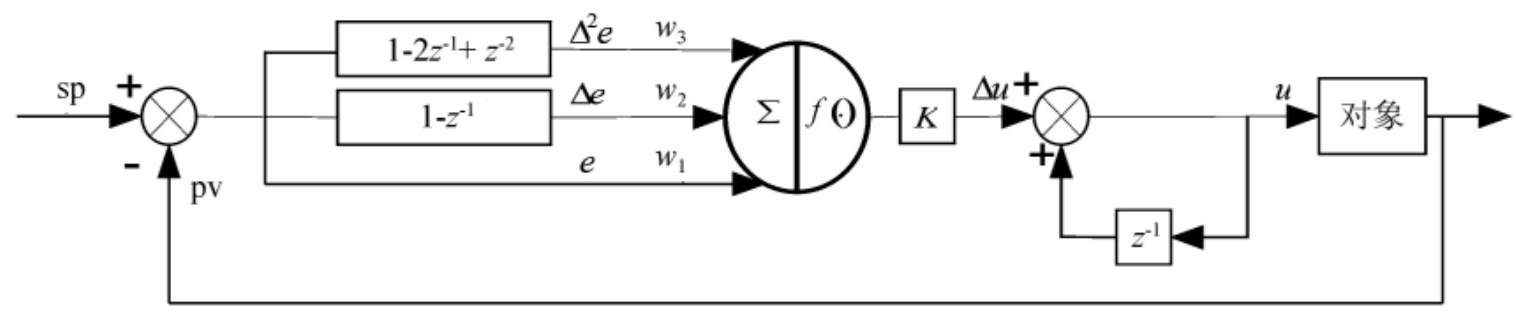

Fig. 1 Structure flow of single - neuron PID controller

In Figure 3, $\omega_{1}, \omega_{2}, \omega_{3}$ is the weighted coefficient, which can be adjusted by the ability of the neuron's self-learning. $\mathrm{K}$ is the neuron's adjustable ratio coefficient. The single neuron adaptive controller realized the adaptive and organizing function by adjusting the weighting coefficients. The adjustment of weight coefficient can adopt different learning rules, and thus constitute different control algorithms. In the experimental project of virtual laboratory, the advanced algorithms are modular, and the sub VI is constructed to be called by other experimental modules. And the realization of the single neuron PID controller will be the use of LabVIEW another way of calling. LabVIEW in the dynamic link library call was achieved through the CLF (Library Function Call) node. In the course of implementation of the single neuron PID controller, single neuron PID control algorithm was coded in the integrated environment of $\mathrm{VC}++$, including set corresponding function name, input and output parameters and return type etc.. Then the generated source code files are compiled, and generate DLL files. So you can call these functions directly in LabVIEW. Then, the $\omega_{1}$, $\omega_{2}, \omega_{3}$ and adjustable ratio coefficient $\mathrm{K}$ are initialized in the main program flow. Figure 2 shows a single neuron PID control algorithm flow chart. Figure 3 shows the VI single_n. flow chart.

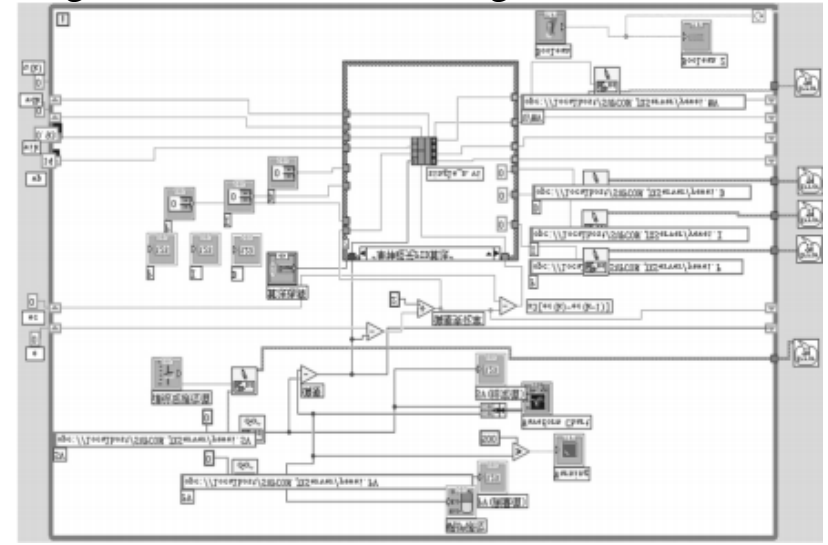

Fig.2 Program flow of single-neuron PID

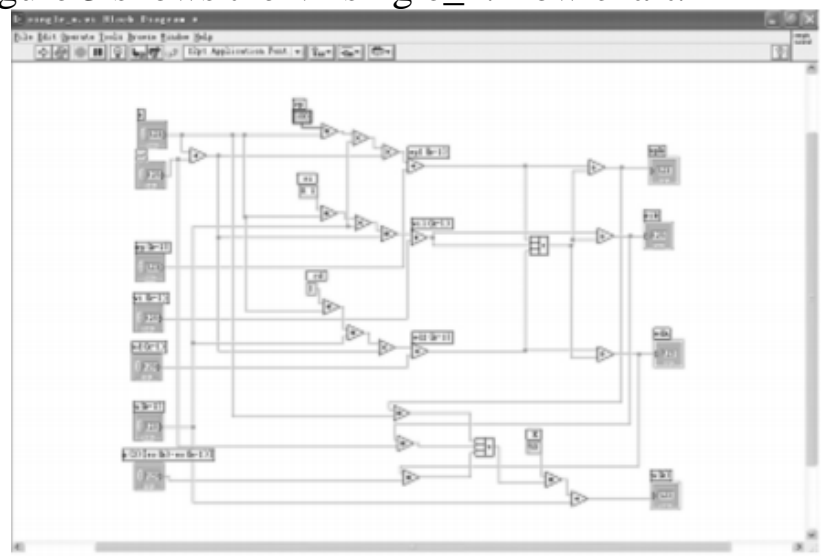

Fig. 3 Program flow of single_n. vi

In the experiment, the controlled object is put into the implementation of step input. That is to set the boiler level from $200 \mathrm{~mm}$ up to $250 \mathrm{~mm}$. The experiment is repeated debugging. The paper selected the appropriate adjustable coefficient, learning rate and other control parameters: $\mathrm{K}=50.0$, $\mathrm{Cp}=100.0, \mathrm{Ci}=0.1, \mathrm{Cd}=1.0$, ts $=2 \mathrm{~s}$. After the experiment, the level of the liquid level is stable. The local end of the single neuron PID controller level response curve shown in Figure 4, we can see that the system response fast, and no overshoot. At the same time, when the external disturbance is added to the system, it is able to return to steady state quickly without oscillation. The experimental results show that the single neuron PID controller in this paper has the ability to be adaptive to the time-varying and large time delay of the system, and it has better dynamic and static performance and robustness. 


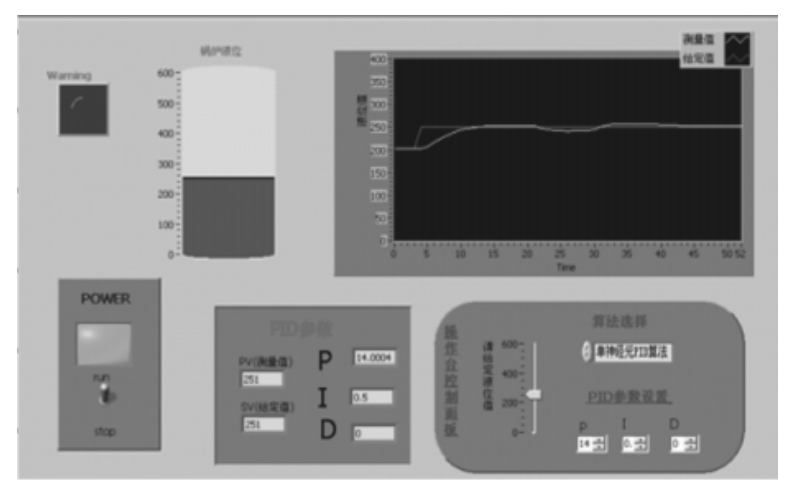

Fig. 4 The local operation curve of single-neuron PID controller

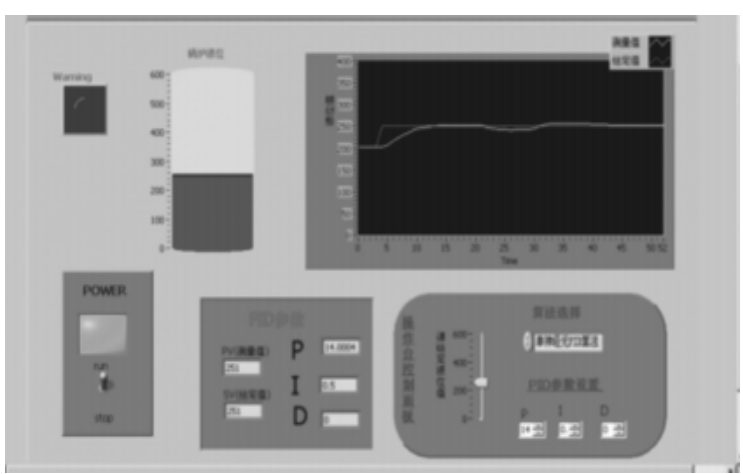

Fig. 5 The operation curve of single-neuron PID controller of the remote-clien

At the same time, the system can achieve remote control experiments on the Internet by virtual laboratory remote client. Because the virtual lab system is based on the virtual experiment server located at the center of the central server, which is responsible for the management and scheduling of each experiment module and the field experiment instrument. On the one hand, the system receive the Web server through the remote client's instructions, and call the relevant experimental module to experiment and download the experimental parameters; On the other hand, the system collected field control end of the experimental data through the interface of OPC server DataSocket protocol, and transmitted in real time to the remote client. The experimental results was shown in Figure 5. The remote client operating curve and the local end is basically the same, the delay is less than $1 \mathrm{~s}$. It is proved that the virtual network laboratory can achieve the basic goal of the network based remote experiment.

\section{Summary}

This paper introduces the overall structure and experimental modules of the virtual network lab. This paper focuses on the design of a 300X - DCS JX as the control system. Boiler process control equipment is used as the controlled object. Single neuron PID is used as the control algorithm of the experimental system. The system was tested on remote synchronization through the Internet. The experiment results show that the results of the remote client experiment and the running results of the local experimental system are consistent. And the delay time is less than $1 \mathrm{~s}$. The function of remote synchronous experiment is realized. At the same time, it proves that the control algorithm has good control effect, which proves the availability of the virtual network lab and the good operation performance.

\section{References}

[1] Luo J, Lei Y, Huang H, et al. Remote experimental platform for motor based on LabVIEW[J]. Experimental Technology \& Management, 2014.

[2] Cao J C, Zhu Y P, Mu X U, et al. Remote Fiber-optics Experiment Platform Based on LabVIEW Technique[J]. Research \& Exploration in Laboratory, 2013.

[3] Gao R J, Zheng Y D, Xiao-Dong L I, et al. Design of anti-lock brake remote experimental system based on LabVIEW[J]. Laboratory Science, 2014.

[4] Luo J, Lei Y, Huang H, et al. Remote experimental platform for motor based on LabVIEW[J]. Experimental Technology \& Management, 2014.

[5] Liu X, Zhang Z J, Li S Z, et al. Research on Real-Time Monitoring and Data Management of Hydraulic Test System Based on LabVIEW[J]. Applied Mechanics \& Materials, 2014, 496-500:1715-1719. 
[6] Li D D. The design of virtual experiment Framework Based on ELVIS[J]. Advanced Materials Research, 2014, 1037:161-164.

[7] Yang J. Study of intelligent testing system of solenoid valve based on LabVIEW[J]. Journal of Digital Convergence, 2013, 11(10).

[8] Lichuan X U, Zhaoyang S U, Liang Y, et al. Design for Experiment of DC Motor Speed Monitoring Based on LabVIEW[J]. Experiment Science \& Technology, 2013.

[9] Dai H T, Chen N I, Fang K, et al. Study of the properties of photovoltaic cells based on LabVIEW[J]. Physics Experimentation, 2014.

[10] Huang Z, Wang X. Development of virtual instrument motor experiment teaching system based on LabVIEW[J]. Journal of Chemical \& Pharmaceutical Research, 2014.

[11] Zhang H X, Yan Y, Zhou H, et al. The Development of Virtual Experiment Platform for Signal Analysis and Processing Based on LabVIEW[J]. Applied Mechanics \& Materials, 2014, 513-517:3241-3244. 